\title{
Icariside II ameliorates myocardial ischemia and reperfusion injury by attenuating inflammation and apoptosis through the regulation of the PI3K/AKT signaling pathway
}

\author{
BING-FENG GUAN $^{1 *}$, XIAO-FENG DAI ${ }^{1 *}$, QI-BIN HUANG $^{1,2}$, DI ZHAO ${ }^{1}$, \\ JIN-LONG SHI ${ }^{1}, \mathrm{CHENG} \mathrm{CHEN}^{1}, \mathrm{YAN} \mathrm{ZHU}^{3}$ and $\mathrm{FEN} \mathrm{AI}^{4}$
}

\author{
${ }^{1}$ Department of Cardiothoracic, The First Affiliated Hospital of Yangtze University, Jingzhou, Hubei 434023; \\ ${ }^{2}$ College of Life Sciences, Hubei University, Wuhan, Hubei 430062; ${ }^{3}$ Department of Oncology, The First Affiliated \\ Hospital of Yangtze University, Jingzhou, Hubei 434023; ${ }^{4}$ Department of Emergency, The Central Hospital of Wuhan, \\ Tongji Medical College, Huazhong University of Science and Technology, Wuhan, Hubei 430014, P.R. China
}

Received November 21, 2019; Accepted June 3, 2020

DOI: $10.3892 / \mathrm{mmr} .2020 .11396$

\begin{abstract}
Icariside II (ICAII) is a bioflavonoid compound which has demonstrated anti-oxidative, anti-inflammatory and anti-apoptotic biological activities. However, to the best of our knowledge, whether ICAII can alleviate myocardial ischemia and reperfusion injury (MIRI) remains unknown. The aim of the present study was to determine whether ICAII exerted a protective effect on MIRI and to investigate the potential underlying mechanism of action. A rat MIRI model was established by ligation of the left anterior descending coronary artery for $30 \mathrm{~min}$, followed by a $24 \mathrm{~h}$ reperfusion. Pretreatment with ICAII with or without a PI3K/AKT inhibitor was administered at the beginning of reperfusion. Morphological and histological analyses were detected using hematoxylin and eosin staining; the infarct size was measured using Evans blue and 2,3,5-triphenyltetrazolium chloride staining; and plasma levels of lactate dehydrogenase
\end{abstract}

Correspondence to: Dr Yan Zhu, Department of Oncology, The First Affiliated Hospital of Yangtze University, 8 Hanghang Road, Shashi, Jingzhou, Hubei 434023, P.R. China

E-mail: 1708695578@qq.com

Dr Fen Ai, Department of Emergency, The Central Hospital of Wuhan, Tongji Medical College, Huazhong University of Science and Technology, 26 Shengli Street, Jiangan, Wuhan, Hubei 430014, P.R. China

E-mail: fenai@163.com

*Contributed equally

Abbreviations: CK-MB, creatine kinase-myocardial band; ICAII, icariside II; IL-6, interleukin-6; LAD, left anterior descending coronary artery; LDH, lactate dehydrogenase; LVEF, left ventricular ejection fraction; MIRI, myocardial ischemia and reperfusion injury; TNF- $\alpha$, tumor necrosis factor- $\alpha$

Key words: icariside II, MIRI, PI3K, AKT
(LDH) and creatine kinase-myocardial band (CK-MB) were analyzed using commercialized assay kits. In addition, the cardiac function was evaluated by echocardiography and the levels of cardiomyocyte apoptosis were determined using a TUNEL staining. The protein expression levels of Bax, Bcl-2, cleaved caspase-3, interleukin-6, tumor necrosis factor- $\alpha$, PI3K, phosphorylated (p)-PI3K, AKT and p-AKT were analyzed using western blotting analysis. ICAII significantly reduced the infarct size, decreased the release of $\mathrm{LDH}$ and CK-MB and improved the cardiac function induced by IR injury. Moreover, ICAII pretreatment significantly inhibited myocardial apoptosis and the inflammatory response. ICAII also upregulated the expression levels of p-PI3K and p-AKT. However, the protective effects of ICAII were abolished by an inhibitor (LY294002) of the PI3K/AKT signaling pathway. In conclusion, the findings of the present study suggested that ICAII may mitigate MIRI by activating the PI3K/AKT signaling pathway.

\section{Introduction}

Acute myocardial infarction (AMI) is the leading cause of mortality and disability worldwide (1). Epidemiological studies have identified risk factors for AMI, including dyslipidemia, diabetes, obesity, hypertension, smoking, sex and genetic variation $(1,2)$. Early prevention and the strict control of related risk factors have become the standard therapies used to prevent AMI (3). AMI results from the abrupt occlusion of coronary arteries, which leads to prolonged myocardial ischemia and irreversible cardiomyocyte death (4). Currently, the most effective way to save apoptotic cardiomyocytes is timely and successful revascularization; however, re-establishing blood flow to the ischemic area has been demonstrated to promote additional myocardial damage, a process referred to as myocardial ischemia and reperfusion injury (MIRI) (5). Moreover, although strategies such as percutaneous coronary intervention, intravenous thrombolysis and coronary artery bypass grafting have markedly limited infarct expansion and decreased in-hospital mortality, the incidence of subsequent 
chronic heart failure has increased gradually (6). Thus, the identification of new drugs that can attenuate MIRI through the aforementioned mechanisms is urgently required.

MIRI involves a complex pathophysiological process, in which inflammation and apoptosis have been recognized as the two characteristics underlying the pathological mechanisms (7). Reperfusion was discovered to trigger a strong inflammatory response, thus recruiting inflammatory cells and releasing inflammatory factors, leading to serious cardiomyocyte damage (8). In addition, several apoptosis-related genes, including Bax and cleaved caspase-3 were identified to be involved in MIRI (9). A previous study suggested that cardiomyocyte apoptosis occurred during the early phase of ischemia and that reperfusion could exacerbate the severity (10). Thus, it is hypothesized that the regulation of the inflammatory response and the concurrent inhibition of cardiomyocyte apoptosis, which are of great clinical significance to the treatment of AMI, may mitigate MIRI.

Icariside II (ICAII) is a ubiquitous bioflavonoid compound derived from Herbaepimedii(11) and used in traditional Chinese medicine. ICAII has demonstrated multiple biological benefits, such as anti-oxidative, anti-inflammatory and anti-apoptotic properties (12). To date, previous studies have predominately focused on the antitumor efficacy of ICAII in different types of cancer, such as lung carcinoma, prostate cancer, melanoma and breast cancer (12), although previous studies have demonstrated that ICAII also exerted a positive effect in the pathological progression of cardiovascular disease. For example, ICAII administration attenuated cardiac remodeling and ameliorated diabetic cardiomyopathy, thereby inhibiting myocardial hypertrophy and myocardial fibrosis $(13,14)$. However, to the best of our knowledge, whether ICAII can alleviate MIRI remains unknown. Interestingly, it has been reported that multiple flavonoid drugs exerted myocardial protective efficiency in MIRI $(10,15)$. Furthermore, a previous study demonstrated that ICAII exerted an anti-inflammatory effect and protected neurons from cerebral IR injury (16). Therefore, the aim of the present study was to determine whether the pretreatment with ICAII mitigated MIRI and to investigate the underlying mechanisms of action.

\section{Materials and methods}

Construction of MIRI model rats. A total of 70 wild-type male Sprague-Dawley rats (specific pathogen-free; weight, 200-250 g; age, 6-7 weeks) were purchased from The Hubei Provincial Center for Disease Control and Prevention. Rats were provided with free access to standard rat chow and water until the time of the experiment, and were housed at $24^{\circ} \mathrm{C}$ and $55 \%$ humidity, with a $12 \mathrm{~h}$-light/dark cycle. Animal health and behaviour were monitored every two days for unexpected pain, poor appetite, weight loss and weakness; no rat died unexpectedly during the experiment ( 3 weeks). The maximum body weight observed in the study was $241.6 \mathrm{~g}$. After the intervention and reperfusion procedures were completed, the rats were anesthetized with $60 \mathrm{mg} / \mathrm{kg}$ pentobarbital sodium intraperitoneally, then sacrificed by air embolization with $0.03 \% \mathrm{CO}_{2}$. Euthanasia was confirmed by loss of vital signs using an electrocardiogram (ECG). All experimental procedures were approved by The Animal Care and Use Committee of Hubei University (Wuhan, China) and performed in accordance with the Institutional Guidelines and the Guide for the Care and Use of Laboratory Animals (National Institutes of Health publication no. 85-23, revised 1996 edition) (17).

The MIRI model rats were established by exerting myocardial ischemia for $30 \mathrm{~min}$ and reperfusion for $24 \mathrm{~h}$. Briefly, animals were fasted $12 \mathrm{~h}$ before surgery, then anesthetized with $60 \mathrm{mg} / \mathrm{kg}$ pentobarbital sodium intraperitoneally and placed in the supine position. A standard lead-II ECG was used to monitor continuously throughout the operation. A median incision in the neck was made to expose the trachea and the rats were artificially ventilated with a small animal respirator at 70 strokes/min. A left thoracotomy was performed and the left arterial descending (LAD) coronary artery was located. A small curved needle with a 6-0 silk suture was then passed through the myocardium beneath the LAD artery and a ligation was performed to block the blood flow. MIRI establishment was considered successful when the ST segment in the lead II was elevated and the regional myocardial surface became pale. Following $30 \mathrm{~min}$ of ischemia, the suture was untied to reperfuse the myocardium for $24 \mathrm{~h}$. After reperfusion, the animals were sacrificed as described and the myocardial specimens were harvested for further analysis.

Experimental design. ICAII (Shanghai Aladdin Biochemical Technology Co., Ltd.) was administered in varying concentrations to determine the optimal drug dose for MIRI improvement. ICAII was dissolved in normal saline containing $0.05 \%$ Tween-80 (Sigma-Aldrich; Merck KGaA) and injected through the tail vein $(300 \mu \mathrm{l})$ at the beginning of the myocardial reperfusion period. Briefly, 30 rats were randomly divided into five groups (6 rats/group): i) A sham-operated ( $\mathrm{SO}$ ) group, in which thoracotomy surgery was performed without ligating the LAD artery; ii) an IR group, in which rats were subjected to LAD artery occlusion for $30 \mathrm{~min}$, followed by reperfusion for $24 \mathrm{~h}$; iii) a $10 \mathrm{mg} / \mathrm{kg}$ ICAII and IR group (ICAII 10 + IR group); iv) a $20 \mathrm{mg} / \mathrm{kg}$ ICAII and IR group (ICAII $20+$ IR group); and v) a $30 \mathrm{mg} / \mathrm{kg}$ ICAII and IR group (ICAII $30+$ IR group). Rats in the last three groups were subjected to LAD artery ligation for $30 \mathrm{~min}$ and then administered the indicated doses of ICAII, followed by a $24 \mathrm{~h}$ myocardial reperfusion.

To investigate the specific underlying protective mechanism of ICAII in MIRI, another experiment was performed, in which rats were randomly divided into four groups ( $n=10$ in each group): i) An SO group; ii) an IR group; iii) an ICAII $20+$ IR group, in which the rat MIRI model was established and $20 \mathrm{mg} / \mathrm{kg}$ ICAII was administered through the tail vein at the beginning of reperfusion; and iv) an ICAII 20 + IR + LY294002 group, treated like the ICAII 20 + IR group, with the addition of $0.3 \mathrm{mg} / \mathrm{kg}$ LY294002 (Sigma-Aldrich; Merck KGaA), a specific inhibitor of the PI3K/AKT signaling pathway. LY294002 was intraperitoneally injected into the abdomen after the LAD artery was untied. An equal volume of DMSO $(0.3 \mathrm{mg} / \mathrm{ml}$; Shanghai Aladdin Biotechnology Technology Co., Ltd.) was similarly administered to the rats in the three groups which did not receive LY294002 treatment.

Histological examination. At the end of the experiment, the left ventricle from each rat was harvested and immersed in a $4 \%$ paraformaldehyde solution for $24 \mathrm{~h}$ at room temperature. 
After fixation, the specimens were embedded in paraffin and the samples were cut into $5-\mu \mathrm{m}$ thick sections on glass slides. Hematoxylin and eosin (H\&E) staining was then performed; sections were stained with hematoxylin for $15 \mathrm{~min}$ at $25^{\circ} \mathrm{C}$ and eosin for $5 \mathrm{~min}$ at $25^{\circ} \mathrm{C}$. The morphology and structure of the myocardial tissue were observed under a light microscope (magnification, $\mathrm{x} 200$ ).

To quantify the severity of the myocardial injury, five randomly selected fields from each group were chosen and scored (damage score) according to the standards described in a previous study (10): i) No injury, 0; ii) interstitial edema and focal necrosis (mild injury), 1; iii) cardiomyocyte swelling and necrosis (moderate injury), 2; iv) formation of necrotic contraction bands and inflammatory cell enrichment (severe injury), 3; and v) expanded necrosis of contraction bands, inflammatory cell infiltration and hemorrhage (highly severe injury), 4.

Infarct size assessment. The size of the myocardial infarct was determined by Evans blue and 2,3,5-triphenyltetrazolium chloride (TTC) staining, as previously described (10) (both from Sigma-Aldrich; Merck KGaA). Masson's trichrome staining was not used in the present study as the experimental design did not allow for the formation of collagen fibers in the myocardial tissue (18). At the end of the experiment (after $24 \mathrm{~h}$ reperfusion and prior to euthanasia), 5 rats were anaesthetized with $60 \mathrm{mg} / \mathrm{kg}$ pentobarbital sodium intraperitoneally. The LAD was re-ligated at the same location and $1 \mathrm{ml}$ Evans blue solution (2\%) was rapidly injected into the jugular vein. The rats were subsequently euthanized as described above and the heart was then removed, rinsed with an ice-cold saline solution and incubated at $-80^{\circ} \mathrm{C}$ for $8 \mathrm{~min}$. The frozen heart was cut into $\sim 2-\mathrm{mm}$ longitudinal sections and incubated with $1 \%$ TTC solution at $37^{\circ} \mathrm{C}$ for $30 \mathrm{~min}$, followed by fixation with $4 \%$ paraformaldehyde for $24 \mathrm{~h}$ at room temperature. The white infarct area and total left ventricular area at the papillary muscle plane of the heart were measured using a digital camera (Nikon Corporation) and Photoshop CS6 software (Adobe Systems, Inc.). The relative myocardial infarct size (\%) was calculated using the following equation: (Infarct area/the total left ventricular area) x100.

Measurement of myocardial injury markers. Myocardial enzymes are released when cardiomyocytes are seriously damaged, with their levels representing the severity of the myocardial injury (7). Before the injection of Evans blue solution, $2 \mathrm{ml}$ blood samples were collected from the jugular vein and centrifuged at $1,006 \mathrm{x}$ g for $10 \mathrm{~min}$ at $4^{\circ} \mathrm{C}$ to obtain plasma. Subsequently, commercially available biochemical kits (Nanjing Jiancheng Bioengineering Institute) were used according to the manufacturers' instructions to detect the levels of lactate dehydrogenase (LDH; cat. no. A020-1-2) and creatine kinase-myocardial band (CK-MB; cat. no. H197).

Examination of cardiac function. Ultrasonic cardiograms were performed to determine the systolic function of the heart following IR injury using the MyLab 30CV ultrasound system (Esaote SpA). Briefly, following the $24 \mathrm{~h}$ myocardium reperfusion, 5 rats were anesthetized by $1.5-2 \%$ isoflurane inhalation (induction 2-3\%), then placed in the supine position. Ultrasound images were observed from the parasternal short axis at the level of the mid-papillary muscle from $\geq 3$ separate cardiac cycles. Left ventricular ejection fraction (LVEF) was measured to determine cardiac function. Following the ultrasound, the rats were anesthetized with $60 \mathrm{mg} / \mathrm{kg}$ sodium pentobarbital, and blood was collected as described above, prior to euthanasia by air embolization as previously described.

Assessment of myocardial apoptosis. IR-induced apoptosis was evaluated using TUNEL staining. Briefly, paraffin-embedded myocardial specimens were cut into $5-\mu \mathrm{m}$-thick sections in the direction of the perpendicular myocardial fibers. The samples were then fixed with $4 \%$ paraformaldehyde for $24 \mathrm{~h}$ at $25^{\circ} \mathrm{C}$. After deparaffinization with dimethylbenzene at $60^{\circ} \mathrm{C}$ and rehydration with a descending alcohol series, TUNEL staining was performed according to the manufacturer's protocol using an In Situ Cell Death Detection kit (cat. no. 11684817910; Roche Diagnostics). Briefly, the sections were stained with TUNEL reagent for $1 \mathrm{~h}$ at $37^{\circ} \mathrm{C}$ and cell nuclei were counterstained at $25^{\circ} \mathrm{C}$ for $10 \mathrm{sec}$ with $3 \%$ ethyl green, then mounted in 50-100 $\mu \mathrm{l}$ Permountä mounting medium. Subsequently, $\geq 5$ randomly selected high-power fields of view of each slice were selected to count the number of apoptotic cardiomyocytes using a fluorescent microscope (magnification, x400). The nuclei of the apoptotic TUNEL-positive cells and normal cells were stained brown and blue, respectively. The apoptotic index (AI; \%) was calculated using the following equation: Ratio of TUNEL-positive cells to total cardiomyocytes.

Western blotting. Total protein was extracted from myocardial samples using RIPA lysis buffer (Beyotime Institute of Biotechnology), supplemented with $50 \mathrm{mM}$ Tris, $150 \mathrm{mM} \mathrm{NaCl}$, $1 \%$ Triton X-100, $1 \%$ sodium deoxycholate, $0.1 \%$ SDS, $2 \mathrm{mM}$ sodium pyrophosphate, $1 \mathrm{mM}$ EDTA and $100 \mathrm{mM}$ PMSF. Total protein was quantified using a bicinchoninic acid protein assay (Beyotime Institute of Biotechnology) and $36 \mu \mathrm{g}$ protein was separated via $10 \%$ SDS-PAGE. The separated proteins were subsequently electrotransferred onto PVDF membranes and blocked with $5 \%$ non-fat dried milk at room temperature for $2 \mathrm{~h}$ to prevent nonspecific binding to the membranes. Then, the membranes were incubated with the following primary antibodies overnight at $4^{\circ} \mathrm{C}$ (all diluted 1:1,000): Anti-Bax (Abcam; cat. no. ab32503), anti-Bcl-2 (Abcam; cat. no. ab196495), anti-cleaved caspase-3 (Abcam; cat. no. ab49822), anti-interleukin (IL)-6 (Abcam; cat. no. ab9324), anti-tumor necrosis factor (TNF)- $\alpha$ (Abcam; cat. no. ab6671), anti-PI3K (Cell Signaling Technology, Inc.; cat. no. 4249S), anti-phosphorylated (p)-PI3K (Cell Signaling Technology, Inc. cat. no. 17366), anti-AKT (Abcam; cat. no. ab179463), anti-p-AKT (Abcam; cat. no. ab182651) and anti-GAPDH (Abcam; cat. no. ab181602). Following the primary antibody incubation, the membranes were incubated with either a horseradish peroxidase (HRP)-conjugated anti-rabbit IgG (Boster Biological Technology; cat. no. BA1054; 1:10,000) or an HRP-conjugated anti-mouse IgG (Abcam; cat. no. ab6789; $1: 10,000)$ secondary antibody for $2 \mathrm{~h}$ at $25^{\circ} \mathrm{C}$. The protein bands were visualized using an enhanced chemiluminescence reagent (Thermo Fisher Scientific, Inc.) and analyzed using ImageJ 1.52q software (National Institutes of Health).

Statistical analysis. All statistical analyses were performed using SPSS 19.0 software (IBM Corp.) and data are presented 
A
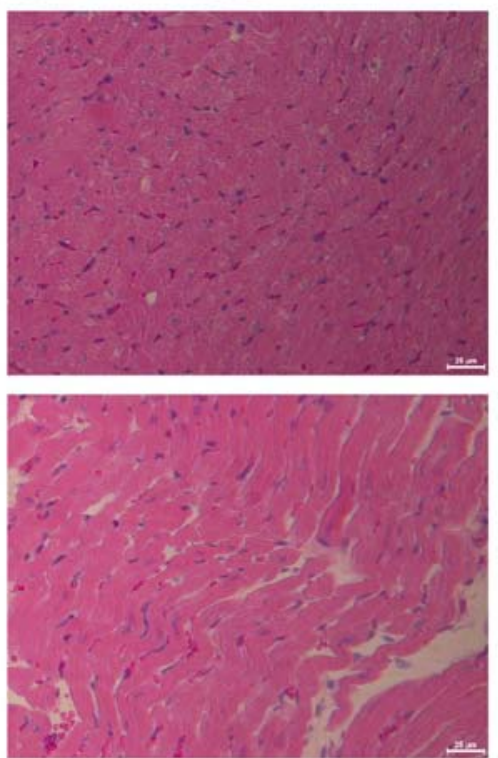

ICAII $20+$ IR
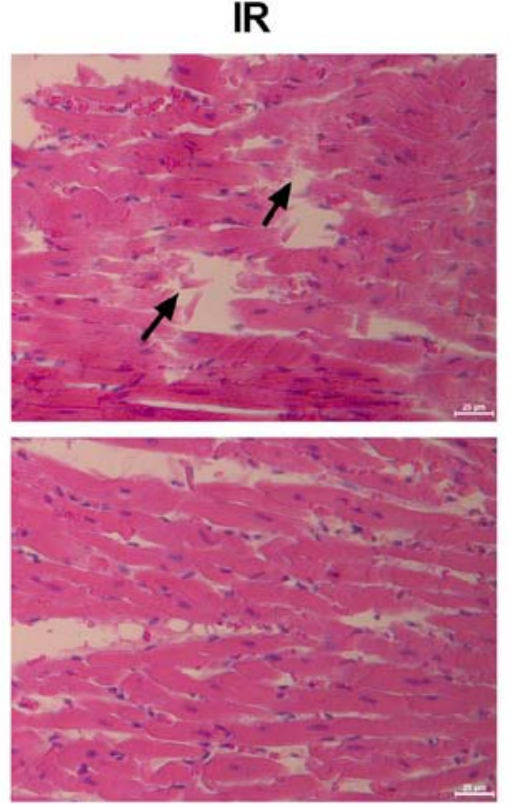

ICAII $30+$ IR

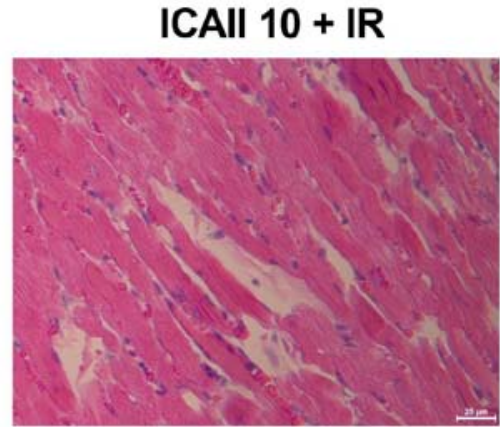

B

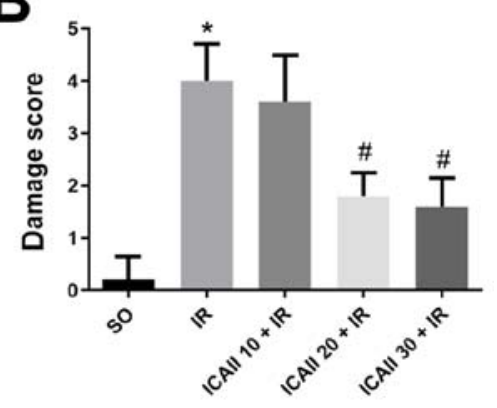

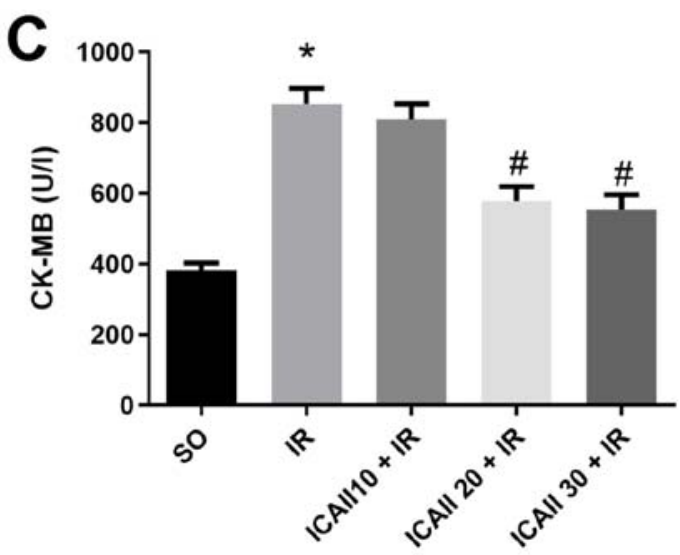

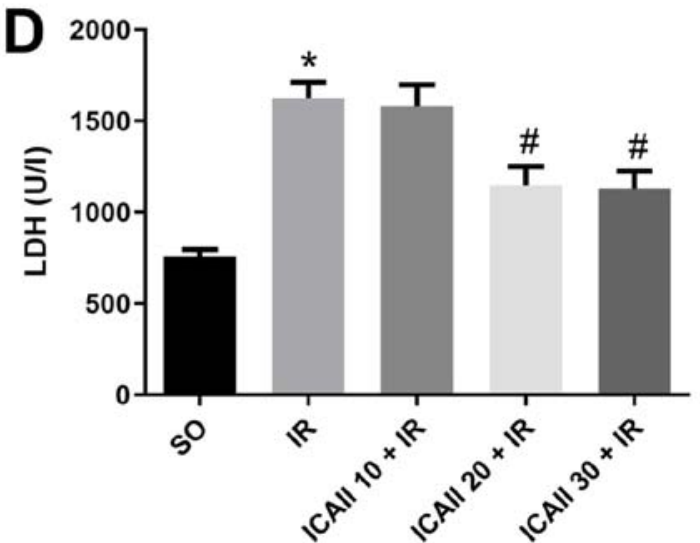

Figure 1. ICAII pretreatment attenuates MIRI. (A) Representative images of hematoxylin and eosin staining in MIRI model rats following the treatment with various doses of ICAII (10, 20 or $30 \mathrm{mg} / \mathrm{kg}$ ). Magnification, x200; scale bar, 25- $\mu \mathrm{m}$. Arrows indicate lesions and myocardial fiber rupture. (B) Damage scores of the severity of myocardial injury from part (A). Serum levels of (C) CK-MB and (D) LDH in each group. $\mathrm{n}=6$. Data are presented as the mean $\pm \mathrm{SD}$. ${ }^{*} \mathrm{P}<0.05$ vs. SO group; "P<0.05 vs. IR group. MIRI, myocardial ischemia and reperfusion injury; ICAII 10/20/30, 10/20/30 mg/kg icariside II; SO, sham-operated; CK-MB, creatine kinase-myocardial band; LDH, lactate dehydrogenase.

as the mean \pm SD of 5 independent repeats. A one-way ANOVA followed by a Tukey's post hoc test was used for multiple comparisons. $\mathrm{P}<0.05$ was considered to indicate a statistically significant difference.

\section{Results}

ICAII attenuates the morphological damage and mitigates MIRI. Myocardial fibres were intact and arranged regularly in the SO group, and no necrosis was observed (Fig. 1A). However, compared with the SO group, the myocardial fibres were disordered and partially ruptured in the IR group, and interstitial edema and inflammatory cell infiltration were observed. By contrast, myocardial damage was less severe following ICAII pretreatment at all doses, and the damage score was significantly decreased in the ICAII $20+$ IR and ICAII 30 + IR groups compared with the IR group. Notably, the cardiomyocyte structure and morphology remained normal in the ICAII $20+$ IR and ICAII $30+$ IR groups, with no significant differences identified in the damage score between these two groups (Fig. 1B).

The levels of myocardial enzymes were also analyzed in the different groups. Serum levels of LDH and CK-MB were significantly increased in the IR group compared with the SO group (Fig. 1C and D). However, only the groups pretreated with 20 and $30 \mathrm{mg} / \mathrm{kg}$ ICAII exhibited significantly decreased enzyme levels compared with the IR group. No significant differences were identified in the levels of the myocardial enzymes between the ICAII 20 + IR and ICAII 30 + IR groups. As a result, $20 \mathrm{mg} / \mathrm{kg}$ was considered as the optimal dose to alleviate myocardial damage.

ICAII pretreatment decreases infarct size and improves cardiac function. Sections of the papillary muscle plane of the 
A

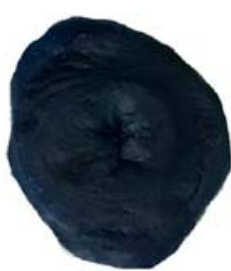

so

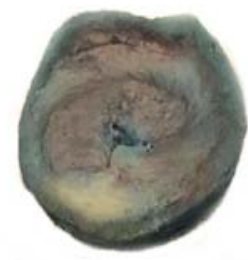

ICAII 20 + IR

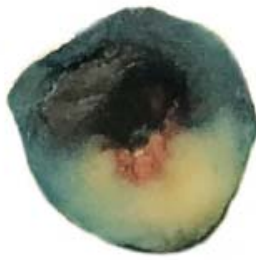

IR

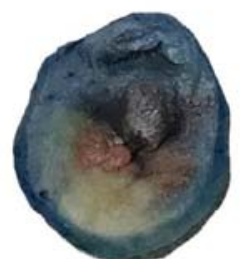

ICAII 20 + IR + LY294002
B

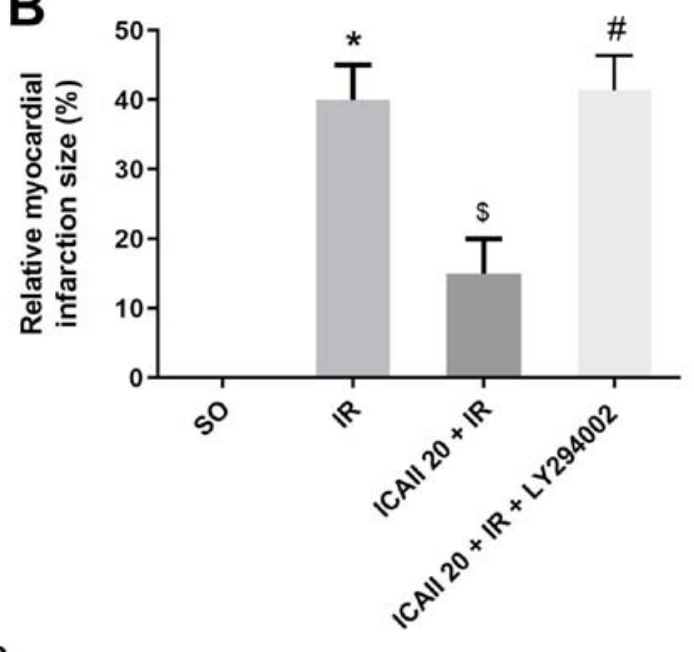

C so
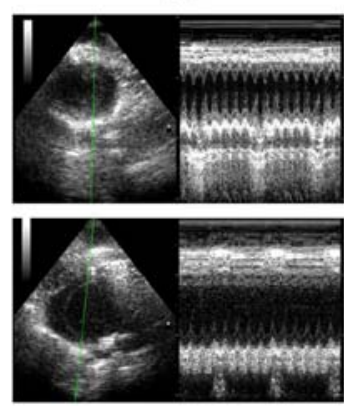

ICAII $20+$ IR
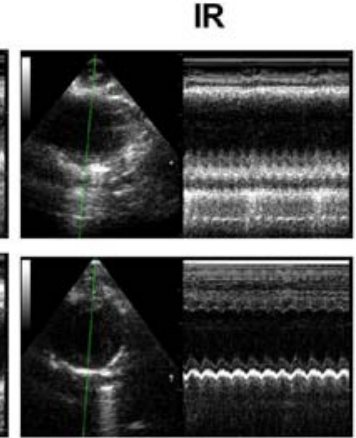

ICAII $20+$ IR + LY294002
D

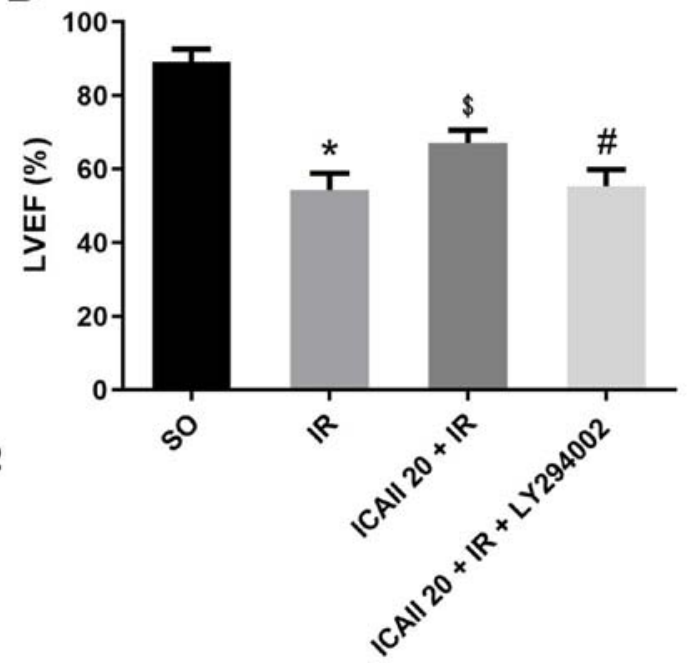

Figure 2. ICAII pretreatment restricts myocardial infarct area and improves cardiac function, while LY294002 partially counteracts this beneficial impact. (A) Evans blue and 2,3,5-triphenyltetrazolium chloride stained samples demonstrating the infarct area (white) and risk area (red) in different groups. (B) Relative myocardial infarction size was calculated from part (A). (C) Representative images of echocardiography. (D) LVEF of each group. $\mathrm{n}=5$. * $\mathrm{P}<0.05$ vs. SO group; ${ }^{~} \mathrm{P}<0.05$ vs. IR group; ${ }^{\prime} \mathrm{P}<0.05$ vs. the ICAII $20+$ IR group. IR, ischemia-reperfusion; ICAII $20,20 \mathrm{mg} / \mathrm{kg}$ icariside II; SO, sham-operated; LVEF, left ventricular ejection fraction.

heart from each group were selected to compare the myocardial infarct size; ICAII pretreatment notably decreased the IR-related infarct size (Fig. 2A and B). Notably, the cotreatment with ICAII and LY294002 significantly counteracted the beneficial effect of ICAII pretreatment. Echocardiography data revealed similar results (Fig. 2C and D); IR injury resulted in the significant degeneration of cardiac function compared with the SO group. However, compared with the IR group, ICAII administration improved LVEF by $19.4 \%$. Notably, the cotreatment with ICAII and LY294002 abolished the improvement in cardiac function observed with ICAII treatment alone.

ICAII pretreatment alleviates IR-induced inflammatory responses. The expression levels of IL-6 and TNF- $\alpha$ in the myocardial tissue were analyzed using western blotting. The expression levels of IL-6 and TNF- $\alpha$ were significantly upregulated following IR injury compared with the SO group (Fig. 3A and B). However, the pretreatment with ICAII significantly downregulated the protein expression levels of IL-6 and TNF- $\alpha$ in the ICAII $20+$ IR group compared with the IR group. By contrast, the anti-inflammatory effect of ICAII was partially suppressed when LY294002 was also administered at the same time.

ICAII pretreatment ameliorates IR-induced myocardial apoptosis. Few TUNEL-positive cells were identified in the SO group, whereas the apoptotic rate of cardiomyocytes was significantly increased following IR injury (Fig. 4A and B). ICAII pretreatment significantly decreased the AI compared with the IR group. However, LY294002 treatment significantly inhibited the effect of ICAII treatment alone on apoptosis.

The expression levels of the proapoptotic protein Bax and the anti-apoptotic protein Bcl-2, as well as cleaved caspase-3, 

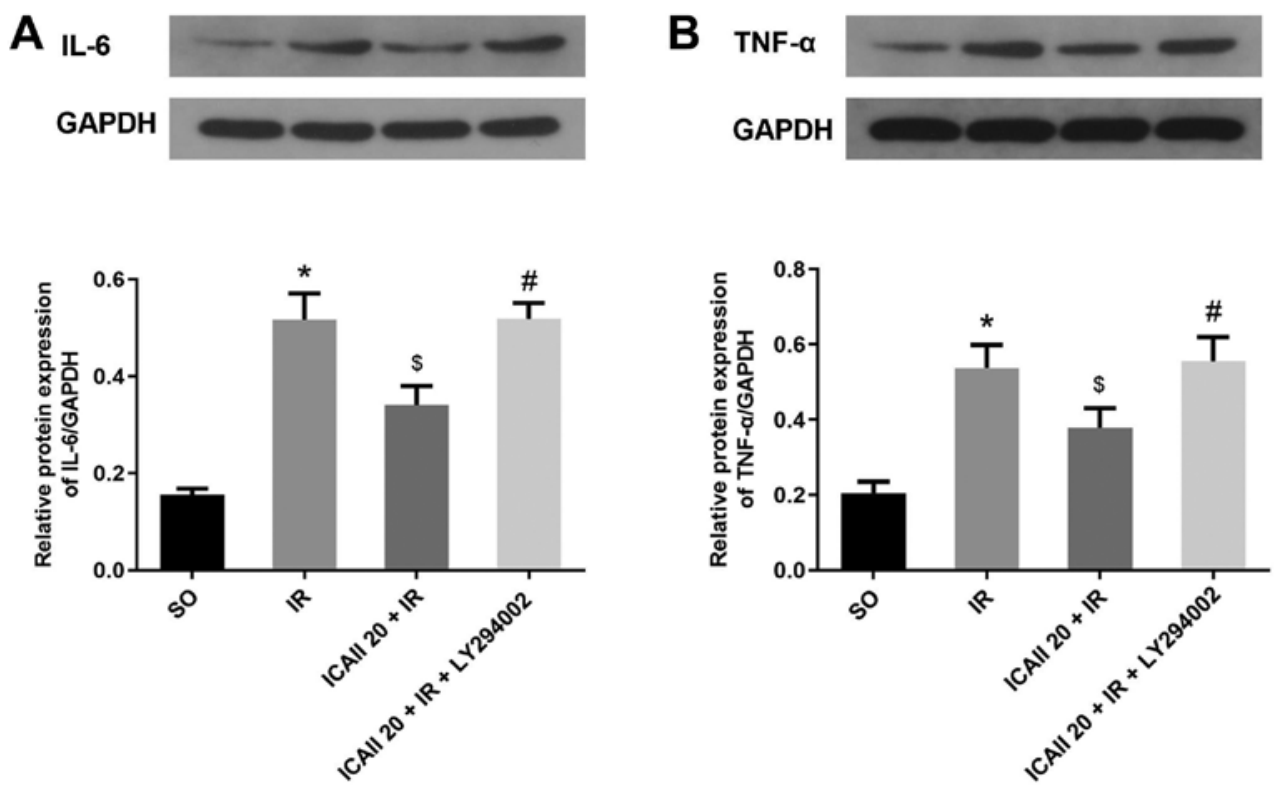

Figure 3. ICAII pretreatment reduces the inflammatory reaction caused by IR injury, while LY294002 significantly counteracts the anti-inflammatory effect of ICAII. Protein expression levels of (A) IL- 6 and (B) TNF- $\alpha$ were determined using western blotting. $n=5 .{ }^{*} \mathrm{P}<0.05$ vs. SO group; ${ }^{\$} \mathrm{P}<0.05$ vs. IR group; ${ }^{*} \mathrm{P}<0.05$ vs. ICAII 20 + IR group. IR, ischemia-reperfusion; ICAII 20, 20 mg/kg icariside II; SO, sham-operated; IL-6, interleukin-6; TNF- $\alpha$, tumor necrosis factor- $\alpha$.

A

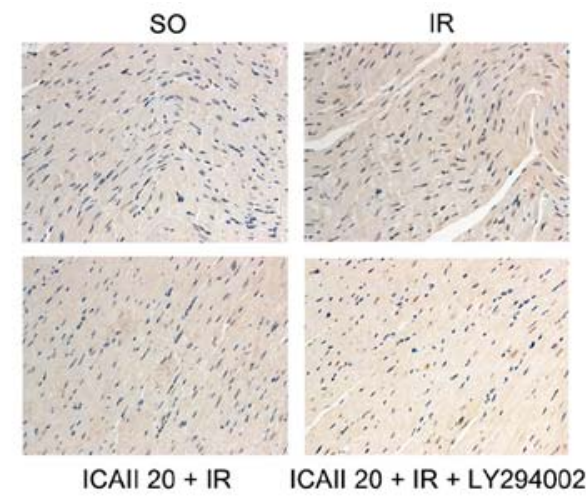

B

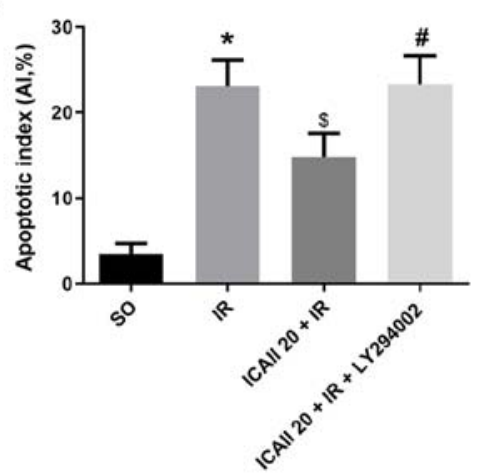

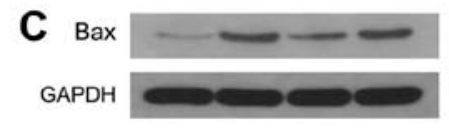

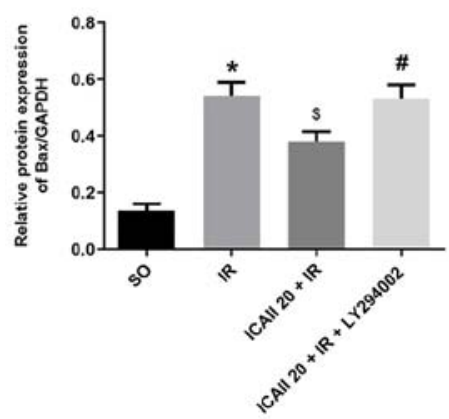

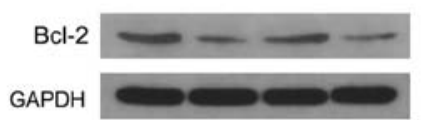

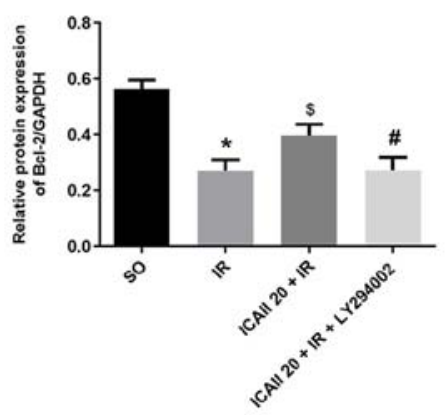

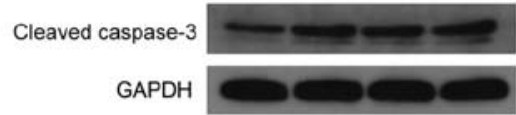

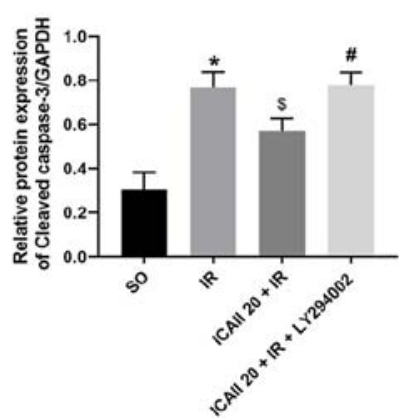

Figure 4. ICAII pretreatment ameliorates IR injury-induced cardiomyocytes apoptosis, while LY294002 partially counteracts the anti-apoptotic effect of ICAII. (A) Representative images of TUNEL stained samples of each group, magnification x400. TUNEL-positive nuclei of apoptotic cardiomyocytes are stained brown and the normal myocardial nuclei are stained blue. (B) Apoptotic indices of different groups were calculated from part (A). (C) Protein expression levels of Bax, $\mathrm{Bcl}-2$ and cleaved caspase- 3 in different groups was determined using western blotting. $\mathrm{n}=5$. ${ }^{*} \mathrm{P}<0.05$ vs. SO group; ${ }^{\text {S }} \mathrm{P}<0.05$ vs. IR group; ${ }^{\text {"P }}<<0.05$ vs. ICAII 20 + IR group. IR, ischemia-reperfusion; ICAII $20,20 \mathrm{mg} / \mathrm{kg}$ icariside II; SO, sham-operated.

were also analyzed. IR injury significantly upregulated the expression levels of Bax and cleaved caspase-3, whilst downregulating the expression levels of Bcl-2 compared with the SO group (Fig. 4C). However, ICAII treatment significantly 

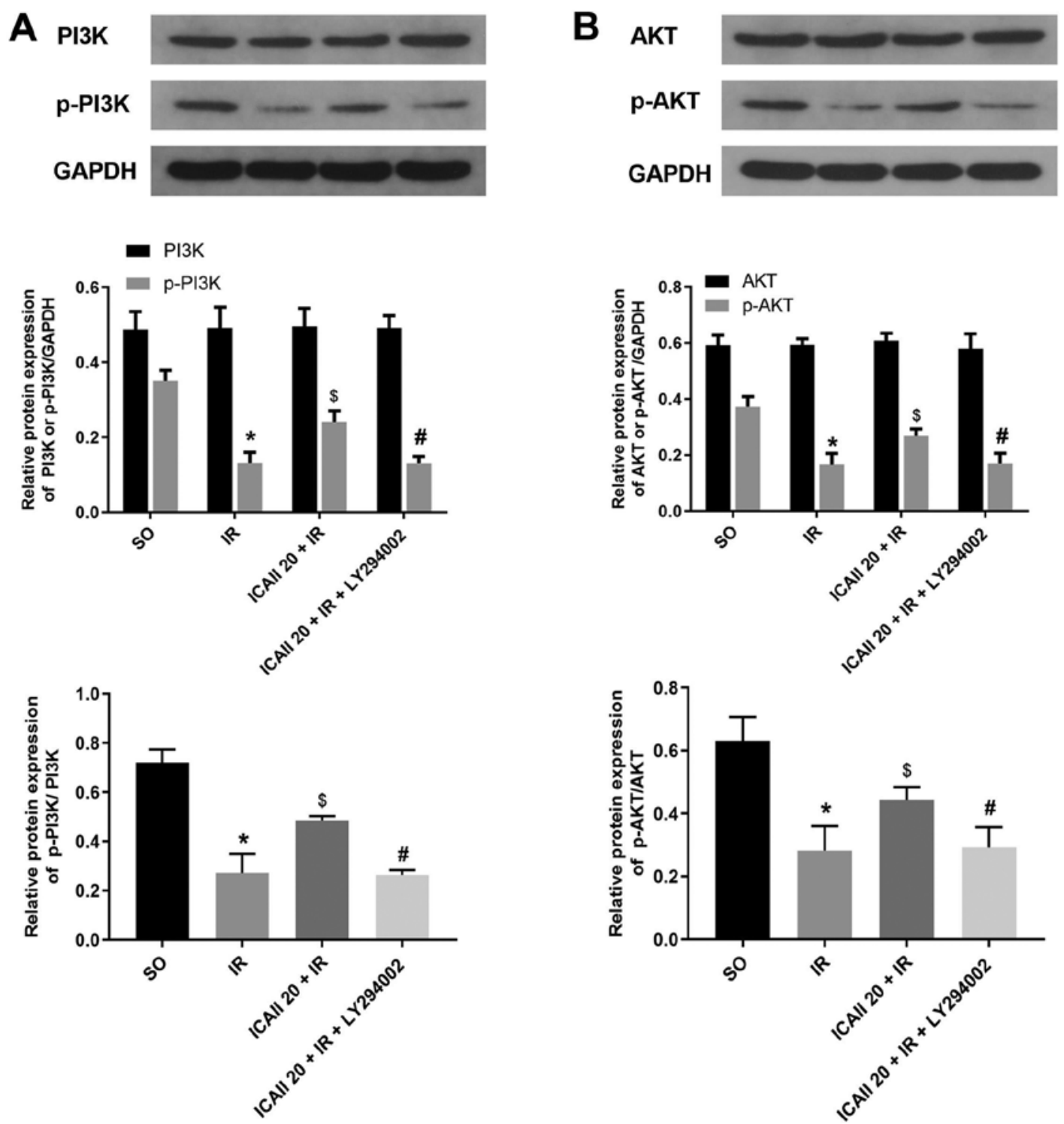

Figure 5. ICAII pretreatment upregulates the expression levels of p-PI3K and p-AKT following IR injury, while LY294002 abolishes this beneficial effect. Protein expression levels of (A) p-PI3K, total PI3K and p-PI3K/PI3K ratio and (B) p-AKT, total AKT and the p-AKT/AKT ratio were determined using western blotting. $\mathrm{n}=5$. ${ }^{\mathrm{P}} \mathrm{P}<0.05$ vs. SO group; ${ }^{\$ \mathrm{P}}<0.05$ vs. IR group; ${ }^{\text {P }}<0.05$ vs. ICAII $20+$ IR group. IR, ischemia-reperfusion; ICAII 20, $20 \mathrm{mg} / \mathrm{kg}$ icariside II; SO, sham-operated; p-, phosphorylated.

downregulated the expression levels of Bax and cleaved caspase-3, while upregulating the expression levels of Bcl-2, compared with the IR group. Notably, the cotreatment with ICAII and LY294002 significantly reversed the trend observed following ICAII treatment alone. These findings suggested that ICAII may prevent IR-induced myocardial apoptosis, while LY294002 may partially inhibit this anti-apoptotic effect.

ICAII pretreatment activates the PI3K/AKT signaling pathway. To further investigate the specific underlying mechanisms of ICAII in alleviating IR injury, the expression levels of molecules involved in the PI3K/AKT signaling pathway were determined. IR injury significantly downregulated the expression levels of p-PI3K and p-AKT compared with the SO group (Fig. 5A and B). However, the pretreatment with ICAII at the onset of reperfusion significantly upregulated the expression levels of p-PI3K and p-AKT compared with the IR group. Conversely, LY294002 treatment significantly reversed the upregulation of $\mathrm{p}-\mathrm{PI} 3 \mathrm{~K}$ and $\mathrm{p}-\mathrm{AKT}$ expression levels promoted by ICAII pretreatment alone. In addition, no significant differences were observed in the expression levels of PI3K and AKT between the different groups. Notably, the ratio of p-PI3K/PI3K and p-AKT/AKT exhibited a similar trend to the expression levels of the phosphorylated proteins. Altogether, these observations indicated that ICAII may activate the PI3K/AKT signaling pathway following IR injury.

\section{Discussion}

Accumulating evidence has suggested that ICAII may serve a beneficial impact in various types of disease, such as Alzheimer's disease, osteoporosis, stroke, breast cancer and leukemia, possibly due to its multiple bioactivities, including antioxidative, anti-inflammatory and anti-apoptotic properties (19). Recent studies have reported that ICAII may also be beneficial for cardiovascular disease; for example, 
in a rat model of spontaneous hypertension, Wu et al (13) demonstrated that the intra-gastric administration of ICAII $(4,8$ or $16 \mathrm{mg} / \mathrm{kg})$ significantly decreased the blood pressure, promoted heart functional recovery and improved ventricular remodeling. Furthermore, mechanistically, ICAII inhibited myocardial apoptosis and decreased the generation of reactive oxygen species (13). Fu et al (20) also identified that the pretreatment with ICAII at the same dose improved myocardial fibrosis through anti-inflammatory mechanisms in the same animal model (spontaneous hypertension). In addition, other studies have indicated that ICAII may serve a positive role during IR injury; for instance, in a rat model of cerebral IR injury, Deng et al (16) demonstrated that 10 or $30 \mathrm{mg} / \mathrm{kg}$ ICAII pretreatment protected neurons from damage and decreased infarct size by regulating the crucial inflammatory factor $\mathrm{NF}-\kappa \mathrm{B}$. Yan et al (21) also suggested that the oral administration of $20 \mathrm{mg} / \mathrm{kg}$ ICAII before IR markedly inhibited IR-induced leukocyte adhesion and neuronal loss in the hippocampal CA1 region. Thus, the aforementioned studies indicated that ICAII may exhibit anti-inflammatory and anti-apoptotic properties at doses of 4-30 mg/kg. However, to the best of our knowledge, whether ICAII prevented MIRI remained unclear.

In the present study, a dose-response experiment demonstrated that ICAII attenuated MIRI at various doses, as evidenced by the mitigation of morphological damage and a decline in the release of $\mathrm{LDH}$ and CK-MB. Furthermore, no statistical significances were reported in the damage scores and myocardial enzyme levels between the middle and high-dose ICAII groups. Thus, $20 \mathrm{mg} / \mathrm{kg}$ ICAII was selected as the dose to elicit a sufficient protective effect on the myocardium. Indeed, the pretreatment with $20 \mathrm{mg} / \mathrm{kg}$ ICAII markedly improved cardiac function, minimized infarct size, reduced inflammatory reactions and decreased cardiomyocyte apoptosis. Moreover, ICAII administration reversed the downregulated expression levels of $\mathrm{p}-\mathrm{PI} 3 \mathrm{~K}$ and $\mathrm{p}-\mathrm{AKT}$ induced by IR injury. Therefore, to the best of our knowledge, the present study was the first to illustrate the protective effects of ICAII against MIRI. The present findings indicated that ICAII may represent a potential therapeutic strategy for MIRI, partly by activating the PI3K/AKT signaling pathway.

It was previously reported that IR was associated with an inflammatory cascade and neutrophil activation (8). The excessive release of cytokines was discovered to be a crucial event in MIRI (22). In the present study, the expression levels of classical inflammatory factors IL-6 and TNF- $\alpha$ were evaluated. Consistent with previous studies, IR injury induced the release of IL- 6 and TNF- $\alpha$ from the myocardial tissue. However, the administration of ICAII at the beginning of reperfusion significantly downregulated IL-6 and TNF- $\alpha$ expression levels. Apoptosis was reported to be the predominant form of cell death in MIRI, leading to the loss of the myocardium and the deterioration of cardiac function (23). The imbalance between anti-apoptotic and proapoptotic protein expression levels were identified as an important characteristic of cell apoptosis (23). The present study identified that IR injury upregulated the expression levels of Bax and cleaved caspase-3, while downregulating the expression levels of Bcl-2. Additionally, the number of TUNEL-positive cells was significantly increased following
IR injury. However, the pretreatment with ICAII reduced the levels of cell apoptosis and reversed the effects of IR on the expression levels of Bax and Bcl-2 to a large extent. Notably, a previous study also identified a feedback loop between inflammation and apoptosis in the pathological progression of MIRI; apoptosis can be regulated by cytokines such as TNF- $\alpha$ and IL-6 and reciprocally, the induced apoptosis provokes a more severe inflammatory response (24). In the present study, ICAII reversed the inflammation and apoptosis caused by IR injury. Nevertheless, the mechanism through which ICAII exerted a myocardial protective effect requires further investigations.

PI3K and its downstream target serine/threonine kinase AKT belong to a conserved family of signal transduction enzymes involved in multiple cellular biological processes (25). The PI3K/AKT signaling pathway is considered as an endogenous negative-feedback regulatory mechanism that promotes cell survival in response to harmful external stimuli (26). Numerous previous studies have provided ample evidence suggesting that the PI3K/AKT signaling pathway may serve a critical role in the pathological progression of MIRI (27-29). The activated form of AKT, p-AKT, regulates numerous apoptosis-related mediators; for example, once activated, p-AKT acts on intrinsic cell death pathways through Bcl-2 family members (Bcl-2, Bcl-xl, Bax and Bad), as well as extrinsic cell death pathways through caspase family members (cleaved caspase-3, caspase-8 and caspase-9) (30). In addition, $\mathrm{PI} 3 \mathrm{~K} / \mathrm{AKT}$ signaling has also been identified to affect several inflammatory-related regulators, such as high mobility group box $1, \mathrm{TNF}-\alpha, \mathrm{NF}-\kappa \mathrm{B}$ and IL-6 $(10,31)$. ICAII has been reported to affect the PI3K/AKT signaling pathway; Luo et al (32) reported that ICAII promoted the osteogenic differentiation of canine bone marrow-derived stem cells by activating the PI3K/AKT signaling pathway. In addition, ICAII exerted an antitumor effect in melanoma and epidermoid carcinoma cells through the activation of the PI3K/AKT signaling pathway $(33,34)$. In the present study, the pretreatment with ICAII not only alleviated inflammation and inhibited the apoptosis induced by IR injury, but it also upregulated the expression levels of p-PI3K and p-AKT. To further verify the causal relationship between the activation of the PI3K/AKT signaling pathway and ICAII, LY294002 (an inhibitor of the PI3K/AKT signaling pathway) was co-administered at the onset of reperfusion. Interestingly, both the cardioprotective effect of ICAII and the upregulated expression levels of $\mathrm{p}-\mathrm{PI} 3 \mathrm{~K}$ and p-AKT were significantly reversed in the presence of LY294002.

In conclusion, the findings of the present study suggested that the pretreatment with ICAII may alleviate MIRI by activating the PI3K/AKT signaling pathway, thereby attenuating inflammatory reactions and myocardial apoptosis. Nonetheless, the present study had several limitations; for example, in addition to anti-inflammatory and anti-apoptotic bioactivities, ICAII has been discovered to possesses antioxidant and autophagy regulatory properties $(12,35)$. These pharmacological activities may be involved in the underlying protective mechanism of ICAII in MIRI; however, these cardioprotective effects of ICAII were not further investigated in vitro in the present study, thus further investigations are required to determine the involvement of these functions 
of ICAII in MIRI. Moreover, in addition to the PI3K/AKT signaling pathway, several other signaling pathways have been reported to be involved in the pathological process of MIRI. For instance, the activation of the STAT3 signaling pathway and the mTOR signaling pathway alleviated myocardial IR injury through suppressing cell apoptosis $(36,37)$; and the activation of the toll-like receptor $4 / \mathrm{NF}-\kappa \mathrm{B}$ and mitogen activated protein kinase signaling pathways exacerbated IR injury through facilitating inflammatory reactions and oxidative stress $(38,39)$. In addition, hypoxia-inducible factor-1 $\alpha / \mathrm{Bcl}$-2-interacting-protein 3-induced autophagy was discovered to serve a protective role during MIRI (40), while glycogen synthase kinase- $3 \beta$ signaling protected cardiomyocytes from hypoxia-reoxygenation injury by preventing calcium overload (41). Therefore, further studies are warranted to determine whether there are any other signaling pathways involved in the protective effects of ICAII in MIRI. Altogether, the findings of the present study indicated that ICAII may be a promising therapeutic option for the treatment of MIRI.

\section{Acknowledgements}

Not applicable.

\section{Funding}

No funding was received.

\section{Availability of data and materials}

The datasets used and/or analyzed during the current study are available from the corresponding author on reasonable request.

\section{Authors' contributions}

BG, YZ and FA made substantial contributions to the conception and design of the study; $\mathrm{BG}$ and $\mathrm{XD}$ acquired the data; $\mathrm{CC}, \mathrm{QH}, \mathrm{JS}$ and DZ analyzed and interpreted the data; BG and $\mathrm{QH}$ were involved in drafting the manuscript; and $\mathrm{YZ}$ and FA revised the manuscript for critically important intellectual content. All authors read and approved the final manuscript.

\section{Ethics approval and consent to participate}

All experimental procedures were approved by The Animal Care and Use Committee of Hubei University (approval no. 20190210; Wuhan, China) and were performed in accordance with the Institutional Guidelines and Guide for the Care and Use of Laboratory Animals (National Institutes for Health publication no. 85-23, revised 1996 edition).

\section{Patient consent for publication}

Not applicable.

\section{Competing interests}

The authors declare that they have no competing interests.

\section{References}

1. Lee SJ, Lee CK, Kang S, Park I, Kim YH, Kim SK, Hong SP, Bae H, He Y, Kubota Y and Koh GY: Angiopoietin-2 exacerbates cardiac hypoxia and inflammation after myocardial infarction. J Clin Invest 120: 5018-5033, 2018.

2. Corrrell CU, Robinson DG, Schooler NR, Brunette MF, Mueser KT, Rosenheck RA, Marcy P, Addington J, Estroff SE, Robinson $\mathrm{J}$, et al: Cardiometabolic risk in patients with first-episode schizophrenia spectrum disorders: Baseline results from the RAISE-ETP study. JAMA Psychiatry 71: 1350-1363, 2014.

3. Maznyczka AM, Oldroyd KG, McCartney P, McEntegart M and Berry C: The potential use of the index of microcirculatory resistance to guide stratification of patients for adjunctive therapy in acute myocardial infarction. JACC Cardiovasc Interv 12: 951-966, 2019.

4. Kimura T, Tajiri K, Sato A, Sakai S, Wang Z, Yoshida T, Uede T, Hiroe M, Aonuma K, Ieda M and Imanaka-Yoshida K: Tenascin-C accelerates adverse ventricular remodeling after myocardial infarction by modulating macrophage polarization. Cardiovasc Res 115: 614-624, 2018

5. Bi X,Zhang G, Wang X, Nguyen C, May HI,Li X,Al-Hashimi AA, Austin RC, Gillette TG, Fu G, et al: Endoplasmic reticulum chaperone GRP78 protects heart from Ischemia/Reperfusion injury through akt activation. Circ Res 122: 1545-1554, 2018.

6. Shah M, Patil S, Patel B, Agarwal M, Davila CD, Garg L, Agrawal S, Kapur NK and Jorde UP: Causes and predictors of 30-day readmission in patients with acute myocardial infarction and cardiogenic shock. Circ Heart Fail 11: e004310, 2018.

7. Zhou H, Ma Q, Zhu P, Ren J, Reiter RJ and Chen Y: Protective role of melatonin in cardiac ischemia-reperfusion injury: From pathogenesis to targeted therapy. J Pineal Res 64, 2018.

8. Yang Y, Lv J, Jiang S, Ma Z, Wang D, Hu W, Deng C, Fan C, Di S, Sun Y and Yi W: The emerging role of Toll-like receptor 4 in myocardial inflammation. Cell Death Dis 7: e2234, 2016.

9. Zhao D, Feng P, Sun Y, Qin Z, Zhang Z, Tan Y, Gao E, Lau WB, Ma X, Yang J, et al: Cardiac-derived CTRP9 protects against myocardial ischemia/reperfusion injury via calreticulin-dependent inhibition of apoptosis. Cell Death Dis 9: 723, 2018.

10. Li X, Hu X, Wang J, Xu W, Yi C, Ma R and Jiang H: Short-Term hesperidin pre-treatment attenuates rat myocardial Ischemia/Reperfusion injury by inhibiting high mobility group box 1 protein expression via the PI3K/Akt pathway. Cell Physiol Biochem 39: 1850-1862, 2016.

11. Li H, Xu Y,Guan R, Matheu M,LeiH, Tian W, GaoZ,Lin G, Guo Y, Xin $\mathrm{Z}$ and Song W: Icariside II prevents high-glucose-induced injury on human cavernous endothelial cells through Akt-eNOS signaling pathway. Andrology 3: 408-416, 2015.

12. Deng Y, Long L, Wang K, Zhou J, Zeng L, He L and Gong Q: Icariside II, a Broad-Spectrum anti-cancer agent, reverses Beta-Amyloid-Induced cognitive impairment through reducing inflammation and apoptosis in rats. Front Pharmacol 8: 39, 2017.

13. Wu Y, Qian Z, Fu S, Yue Y, Li Y, Sun R, Huang B and Yang D: IcarisideII improves left ventricular remodeling in spontaneously hypertensive rats by inhibiting the ASK1-JNK/p38 signaling pathway. Eur J Pharmacol 819: 68-79, 2018.

14. Yang L, Peng C, Xia J, Zhang W, Tian L, Tian Y, Yang X and Cao Y: Effects of icariside II ameliorates diabetic cardiomyopathy in streptozotocin-induced diabetic rats by activating Akt/NOS/NF-kB signaling. Mol Med Rep 17: 4099-4105, 2018.

15. Rani N, Bharti S, Manchanda M, Nag TC, Ray R, Chauhan SS, Kumari S and Arya DS: Regulation of heat shock proteins 27 and 70, p-Akt/p-eNOS and MAPKs by Naringin Dampens myocardial injury and dysfunction in vivo after ischemia/reperfusion. PLoS One 8: e82577, 2013.

16. Deng Y, Xiong D, Yin C, Liu B, Shi J and Gong Q: Icariside II protects against cerebral ischemia-reperfusion injury in rats via nuclear factor- $\kappa \mathrm{B}$ inhibition and peroxisome proliferatoractivated receptor up-regulation. Neurochem Int 96: 56-61, 2016.

17. Guide for the Care and Use of Laboratory Animals Institute of Laboratory Animal Research. Commission on Life Sciences, National Research Council pp.140, 1996. ISBN: 0-309-58869-3, 6x9.

18. Valle RJ, Mauro AG, Devarakonda T, Marchetti C, He J, Kim E, Filippone S, Das A, Toldo S, Abbate A and Salloum FN: Reperfusion therapy with recombinant human relaxin-2 (Serelaxin) attenuates myocardial infarct size and NLRP3 inflammasome following ischemia/reperfusion injury via eNOS-dependent mechanism. Cardiovasc Res 113: 609-619, 2017. 
19. Zheng Y, Deng Y, Gao JM, Lv C, Lang LH, Shi JS, Yu CY and Gong QH: Icariside II inhibits lipopolysaccharide-induced inflammation and amyloid production in rat astrocytes by regulating $\mathrm{IKK} / \mathrm{I} \kappa \mathrm{B} / \mathrm{NF}-\kappa \mathrm{B} / \mathrm{BACE} 1$ signaling pathway. Acta Pharmacol Sin 41: 154-162, 2020.

20. Fu S, Li YL, Wu YT, Yue Y, Qian ZQ and Yang DL: Icariside II attenuates myocardial fibrosis by inhibiting nuclear factor- $\kappa \mathrm{B}$ and the TGF- $\beta 1 /$ Smad 2 signaling pathway in spontaneously hypertensive rats. Biomed Pharmacother 100: 64-71, 2018.

21. Yan BY, Pan CS, Mao XW, Yang L, Liu YY, Yan L, Mu HN Wang CS, Sun K, Liao FL, et al: Icariside II improves cerebral microcirculatory disturbance and alleviates hippocampal injury in gerbils after ischemia-reperfusion. Brain Res 1573: 63-73, 2014.

22. Liu JY, Shang J, Mu XD and Gao ZY: Protective effect of down-regulated microRNA-27a mediating high thoracic epidural block on myocardial ischemia-reperfusion injury in mice through regulating $\mathrm{ABCA} 1$ and $\mathrm{NF}-\mathrm{\kappa} \mathrm{B}$ signaling pathway. Biomed Pharmacother 112: 108606, 2019.

23. Yang J, Guo X, Yang J, Ding JW, Li S, Yang R, Fan ZX and Yang CJ: RP105 protects against apoptosis in ischemia/reperfusion-induced myocardial damage in rats by suppressing TLR4-mediated signaling pathways. Cell Physio Biochem 36: 2137-2148, 2015.

24. Zhang BF, Jiang H, Chen J, Guo X, Li Y, Hu Q and Yang S: Nobiletin ameliorates myocardial ischemia and reperfusion injury by attenuating endoplasmic reticulum stress-associated apoptosis through regulation of the PI3K/AKT signal pathway. Int Immunopharmacol 73: 98-107, 2019.

25. Yu JS and Cui W: Proliferation, survival and metabolism: The role of PI3K/AKT/mTOR signaling in pluripotency and cell fate determination. Development 143: 3050-3060, 2016.

26. Williams DL, Ozment-Skelton T and Li C: Modulation of the phosphoinositide 3-kinase signaling pathway alters host response to sepsis, inflammation, and ischemia/reperfusion injury. Shock 25: 432-439, 2006.

27. Hao YL, Fang HC, Zhao HL, Li XL, Luo Y, Wu BQ, Fu MJ, Liu W, Liang JJ and Chen XH: The role of microRNA-1 targeting of MAPK3 in myocardial ischemia-reperfusion injury in rats undergoing sevoflurane preconditioning via the PI3K/Akt pathway. Am J Physiol Cell Physiol 315: C380-C388, 2018.

28. Liu S, Ai Q, Feng K, Li Y and Liu X: The cardioprotective effect of dihydromyricetin prevents ischemia-reperfusion-induced apoptosis in vivo and in vitro via the PI3K/Akt and HIF-lalpha signaling pathways. Apoptosis 21: 1366-1385, 2016.

29. Li Q, Shen L, Wang Z, Jiang HP and Liu LX: Tanshinone IIA protects against myocardial ischemia reperfusion injury by activating the PI3K/Akt/mTOR signaling pathway. Biomed Pharmacother 84: 106-114, 2016.

30. Zeng C, Jiang W, Zheng R, He C, Li J and Xing J: Cardioprotection of tilianin ameliorates myocardial ischemia-reperfusion injury: Role of the apoptotic signaling pathway. PLoS One 13: e193845, 2018.
31. Pan T, Shi X, Chen H, Chen R, Wu D, Lin Z, Zhang J and Pan J: Correction to: Geniposide suppresses interleukin-1 $\beta$-induced inflammation and apoptosis in rat chondrocytes via the $\mathrm{PI} 3 \mathrm{~K} / \mathrm{Akt} / \mathrm{NF}-\mathrm{\kappa B}$ signaling pathway. Inflammation 42: 404-405, 2019.

32. Luo G, Xu B and Huang Y: Icariside II promotes the osteogenic differentiation of canine bone marrow mesenchymal stem cells via the $\mathrm{PI} 3 \mathrm{~K} / \mathrm{AKT} / \mathrm{mTOR} / \mathrm{S} 6 \mathrm{~K} 1$ signaling pathways. Am J Transl Res 9: 2077-2087, 2017.

33. Wu J, Xu J, Eksioglu EA, Chen X, Zhou J, Fortenbery N, Wei $S$ and Dong J: Icariside II induces apoptosis of melanoma cells through the downregulation of survival pathways. Nutr Cancer 65: 110-117, 2013

34. Wu J, Zuo F, Du J, Wong PF, Qin H and Xu J: Icariside II induces apoptosis via inhibition of the EGFR pathways in A431 human epidermoid carcinoma cells. Mol Med Rep 8: 597-602, 2013.

35. Gao J, Long L, Xu F, Feng L, Liu Y, Shi J and Gong Q: Icariside II, a phosphodiesterase 5 inhibitor, attenuates cerebral ischaemia/reperfusion injury by inhibiting glycogen synthase kinase-3 $\beta$-mediated activation of autophagy. Br J Pharmacol 177: $1434-1452,2020$

36. Cao X, Zhu N, Zhang Y, Chen Y, Zhang J, Li J, Hao P, Gao C and Li L: Y-box protein 1 promotes hypoxia/reoxygenation- or ischemia/reperfusion-induced cardiomyocyte apoptosis via SHP-1-dependent STAT3 inactivation. J Cell Physiol: Jan 22, 2020 doi: 10.1002/jcp.29474 (Online ahead of print).

37. Li CY, Yang P, Jiang YL, Lin Z, Pu YW, Xie LQ, Sun L and Lu D: Ginsenoside Rb1 attenuates cardiomyocyte apoptosis induced by myocardial ischemia reperfusion injury through mTOR signal pathway. Biomed Pharmacother 125: 109913, 2020.

38. Wang L, Liu XH, Chen H, Chen ZY, Weng XD, Qiu T and Liu L: Picroside II protects rat kidney against ischemia/reperfusion-induced oxidative stress and inflammation by the TLR4/NF- $\mathrm{B}$ pathway. Exp Ther Med 9: 1253-1258, 2015.

39. Liu K, Wang F, Wang S, Li WN and Ye Q: Mangiferin attenuates myocardial ischemia-reperfusion injury via MAPK/Nrf-2/HO-1/NF- $\mathrm{KB}$ in vitro and in vivo. Oxid Med Cell Longev 2019: 7285434, 2019.

40. Zhang Y, Liu D, Hu H, Zhang $\mathrm{P}$, Xie R and Cui W: HIF-1 $\alpha /$ BNIP3 signaling pathway-induced-autophagy plays protective role during myocardial ischemia-reperfusion injury. Biomed Pharmacother 120: 109464, 2019.

41. He X, Li S, Fang X and Liao Y: TDCPP protects cardiomyocytes from hypoxia-reoxygenation injury induced apoptosis through mitigating calcium overload and promotion GSK-3 $\beta$ phosphorylation. Regul Toxicol Pharmacol 92: 39-45, 2018.

This work is licensed under a Creative Commons Attribution-NonCommercial-NoDerivatives 4.0 International (CC BY-NC-ND 4.0) License. 